\section{Uso de filtros de veia cava}

Tradicionalmente, filtros de veia cava têm sido indicados para pacientes com TEP aguda, na presença de contraindicação ou de complicação relacionada ao uso de anticoagulantes. (1-3) São também utilizados nas recidivas de TEP, a despeito de anticoagulação adequada, bem como na presença de TEP maciça e nos casos cirúrgicos de embolectomia. ${ }^{(2)}$ Mais recentemente, sobretudo com o advento dos filtros removíveis, sua indicação foi estendida para pacientes acometidos por TVP proximal com alto risco temporário para TEP. ${ }^{(4)}$ Nos últimos anos, devido à maior prevalência de trombos originários das extremidades superiores, filtros também têm sido instalados na veia cava superior. ${ }^{(5)}$

Há apenas um estudo randomizado, controlado, que procura demonstrar a eficácia e a segurança dos $\mathrm{FVCl}$ na prevenção de TEP em pacientes com trombose venosa proximal. (6) Foram implantados $\mathrm{FVCl}$ em 200 pacientes com TVP proximal, sob risco de TEP, enquanto outros 200 indivíduos não utilizaram os filtros. Todos os 400 pacientes foram tratados com heparina e cumarínicos da forma tradicional. Ficou demonstrado que, embora o grupo que utilizou os $\mathrm{FVCl}$ tenha tido menor incidência de TEP, houve um maior número de recidivas de TVP, todavia, sem aumento da mortalidade. Esse mesmo grupo de pesquisadores realizou uma segunda análise, com os mesmos pacientes, estudados 8 anos depois. ${ }^{(7)}$ Ao final do período, além da heparina ministrada na fase aguda, $50 \%$ dos pacientes ainda estavam em uso de cumarínicos. $\mathrm{Na}$ fase inicial,os $\mathrm{FVCl}$ reduziram o risco de TEP. A médio e a longo prazo, os $\mathrm{FVCl}$ foram suficientes para reduzir a ocorrência de TEP, a despeito do aumento na incidência de TVP. 0 maior número de casos de TVP não se traduziu em uma maior incidência de síndrome pós-trombótica. Não houve impacto na redução da mortalidade.

Complicações com uso de filtros de veia cava permanentes são comuns. Complicações precoces incluem a trombose do sítio de punção, assim como a recorrência de TVP e a síndrome pós-trombótica tardias. A oclusão da veia cava inferior ocorre em 22\% dos pacientes após 5 anos e em 33\% após 9 anos, independentemente do uso e do tempo de anticoagulação. ${ }^{(8)}$

0 uso sistemático de filtros de veia cava em nível terapêutico não está indicado para pacientes não selecionados com TVP e candidatos ao uso de anticoagulantes.(A) Devem ser utilizados em pacientes com contraindicação ao uso de anticoagulantes ou que apresentam recorrência apesar do tratamento farmacológico adequado. (C) Pacientes com filtros permanentes, após passada a contraindicação de anticoagulantes, deverão receber essa terapia adicionalmente.

Filtros removíveis devem ser empregados para pacientes com contraindicação temporária ao uso de anticoagulantes, devendo permanecer por aproximadamente 2 semanas.(D)

0 uso de filtros de veia cava deve ser considerado no pré-operatório de pacientes candidatos à cirurgia e acometidos por evento trombótico nos últimos 30 dias, para os quais a anticoagulação precisa ser interrompida..$^{(9,10)}$ Filtros removíveis devem ser considerados na presença de uma contraindicação temporária à anticoagulação.(D)

\section{Referências}

1. Kinney TB. Update on inferior vena cava filters. J Vasc Interv Radiol. 2003;14(4):425-40.

2. Kearon C, Kahn SR, Agnelli G, Goldhaber S, Raskob GE, Comerota AJ, et al. Antithrombotic therapy for venous thromboembolic disease: American College of Chest Physicians Evidence-Based Clinical Practice Guidelines (8th Edition). Chest. 2008;133(6 Suppl):454S-545S.

3. Girard P, Stern JB, Parent F. Medical literature and vena cava filters: so far so weak. Chest. 2002;122(3):963-7.

4. Pacouret G, Alison D, Pottier JM, Bertrand P, Charbonnier B. Free-floating thrombus and embolic risk in patients with angiographically confirmed proximal deep venous thrombosis. A prospective study. Arch lntern Med. 1997;157(3):305-8.

5. Spence LD, Gironta MG, Malde HM, Mickolick CT, Geisinger MA, Dolmatch BL. Acute upper extremity deep venous thrombosis: safety and effectiveness of superior vena caval filters. Radiology. 1999;210(1):53-8.

6. Decousus H, Leizorovicz A, Parent F, Page Y, Tardy B, Girard $P$, et al. A clinical trial of vena caval filters in the prevention of pulmonary embolism in patients with proximal deep-vein thrombosis. Prévention du Risque d'Embolie Pulmonaire par Interruption Cave Study Group. N Engl J Med. 1998;338(7):409-15.

7. PREPIC Study Group. Eight-year follow-up of patients with permanent vena cava filters in the prevention of pulmonary embolism: the PREPIC (Prevention du Risque d'Embolie Pulmonaire par Interruption Cave) randomized study. Circulation. 2005;112(3):416-22.

8. Torbicki A, Perrier A, Konstantinides S, Agnelli G, Galiè $N$, Pruszczyk $P$, et al. Guidelines on the diagnosis and management of acute pulmonary embolism: the Task Force for the Diagnosis and Management of Acute Pulmonary Embolism of the European Society of Cardiology (ESC). Eur Heart J. 2008;29(18):2276-315.

9. Levine MN, Raskob G, Landefeld S, Kearon C. Hemorrhagic complications of anticoagulant treatment. Chest. 1998;114(5 Suppl):511S-523S.

10. Kearon C, Hirsh J. Management of anticoagulation before and after elective surgery. $N$ Engl $\mathrm{J}$ Med. 1997;336(21):1506-11. 